\title{
A spastic paraplegia mouse model reveals REEP1-dependent ER shaping
}

Christian Beetz, ${ }^{1}$ Nicole Koch, ${ }^{2}$ Mukhran Khundadze, ${ }^{3}$ Geraldine Zimmer, ${ }^{3}$ Sandor Nietzsche, ${ }^{4}$ Nicole Hertel, ${ }^{5}$ Antje-Kathrin Huebner, ${ }^{3}$ Rizwan Mumtaz, ${ }^{3}$ Michaela Schweizer, ${ }^{6}$ Elisabeth Dirren, ${ }^{7}$ Kathrin N. Karle, ${ }^{8}$ Andrey Irintchev, ${ }^{9}$ Victoria Alvarez, ${ }^{10}$ Christoph Redies, ${ }^{5}$ Martin Westermann, ${ }^{4}$ Ingo Kurth, ${ }^{3}$ Thomas Deufel, ${ }^{1}$ Michael M. Kessels, ${ }^{2}$ Britta Qualmann, ${ }^{2}$ and Christian A. Hübner ${ }^{3}$

1'nstitute of Clinical Chemistry and Laboratory Diagnostics, 2Institute of Biochemistry I, 3nstitute of Human Genetics, " EElectron Microscopy Center, and

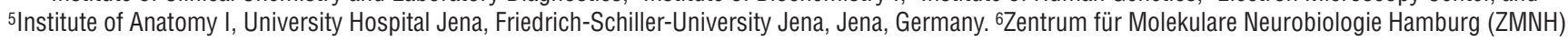
University Hamburg, Hamburg, Germany. Brain Mind Institute, Ecole Polytechnique Fédérale de Lausanne, EPFL, Lausanne, Switzerland.

${ }^{8}$ Department of Neurology and Hertie-Institute for Clinical Brain Research, University of Tübingen, Tübingen, Germany. ${ }^{9}$ Department of Otorhinolaryngology, Jena University Hospital, Friedrich-Schiller-University Jena, Jena, Germany. ${ }^{10 L a b o r a t o r y ~ o f ~ M o l e c u l a r ~ G e n e t i c s ~-~ G e n e t i c ~ U n i t, ~}$ Hospital Universitario Central de Asturias, Oviedo, Spain.

\begin{abstract}
Axonopathies are a group of clinically diverse disorders characterized by the progressive degeneration of the axons of specific neurons. In hereditary spastic paraplegia (HSP), the axons of cortical motor neurons degenerate and cause a spastic movement disorder. HSP is linked to mutations in several loci known collectively as the spastic paraplegia genes (SPGs). We identified a heterozygous receptor accessory protein 1 (REEP1) exon 2 deletion in a patient suffering from the autosomal dominantly inherited HSP variant SPG31. We generated the corresponding mouse model to study the underlying cellular pathology. Mice with heterozygous deletion of exon 2 in Reep1 displayed a gait disorder closely resembling SPG31 in humans. Homozygous exon 2 deletion resulted in the complete loss of REEP1 and a more severe phenotype with earlier onset. At the molecular level, we demonstrated that REEP1 is a neuron-specific, membrane-binding, and membrane curvature-inducing protein that resides in the ER. We further show that Reep1 expression was prominent in cortical motor neurons. In REEP1-deficient mice, these neurons showed reduced complexity of the peripheral ER upon ultrastructural analysis. Our study connects proper neuronal ER architecture to long-term axon survival.
\end{abstract}

\section{Introduction}

Axons are elaborate subcellular structures: once established, they comprise most of the cell's volume (up to $99 \%$ in motor neurons) and account for most of its membrane surface. Yet, the synthesis of structural and functional components largely takes place in the soma (1). As a consequence, lifelong maintenance of axon integrity imposes a great challenge to neurons and requires the concerted action of numerous cellular components and processes. In line with this notion, various genetic defects lead to axon degeneration, while the parent cell bodies and dendrites are spared. Taken together, such axonopathies are among the most common hereditary diseases (2).

A length-dependent degeneration of cortical motor neuron axons results in a spastic gait disorder in hereditary spastic paraplegias (HSPs) (3). With more than 40 spastic paraplegia gene (SPG) loci described and more than 20 genes already identified, HSP is genetically highly heterogeneous (4). HSP is therefore considered a model disease for unraveling the various requirements for long-term axon survival (5). The cellular functions of HSP genes and the molecular pathomechanisms, however, are only beginning to be understood (5-7).

SPG31 represents an autosomal dominant HSP and is caused by mutations in REEP1 (8). Based on the truncating nature of most mutations, a loss-of-function mechanism has been suggested $(9,10)$ but not yet proven. REEP1 belongs to a gene family that consists of two subfamilies in vertebrates (REEP1-REEP4 and REEP5-REEP6) and a single ancestral gene in yeast (YOP1) (11).

Authorship note: Christian Beetz and Nicole Koch contributed equally to this work. Conflict of interest: The authors have declared that no conflict of interest exists. Citation for this article: J Clin Invest. 2013;123(10):4273-4282. doi:10.1172/JCI65665.
REEP proteins contain two hydrophobic regions, and the more C-terminal domain (the "REEP domain") defines the family and is substantially longer than conventional transmembrane domains. Similar regions are found in the evolutionarily unrelated caveolins, reggie/flottilins, and reticulons (12). They have been suggested to insert as hairpins into one leaflet of cellular membranes and to mediate intramembrane homo- and heteromeric interactions $(11,13)$. Hairpin-like membrane insertion has been proposed as a mechanism for curvature generation, curvature stabilization, and/or curvature sensing, specifically in the peripheral ER (14-17). With regard to REEPs, current knowledge is largely based on in vitro assays with REEP5, on overexpression in cell lines, and on genetic manipulation of the yeast ortholog Yop1p (11, 13-16, 18). Loss-of-function approaches in multicellular organisms and detailed characterization of the consequences are not yet available.

In order to unravel the pathophysiology caused by mutations in REEP1 in vivo, we modeled SPG31 in mice. This resulted in REEP1 dose-dependent phenotypes and pathologies closely resembling those seen in HSP patients. At the subcellular level, we observed a reduced complexity of the peripheral ER in cortical motor neurons. Together with in vitro evidence for REEP1 in the induction of positive membrane curvature, our study connects impaired ER shaping to a failure in maintaining long axons.

\section{Results}

Absence of REEP1 exon 2 is associated with a spastic paraplegia phenotype in both bumans and mice. Upon screening HSP patients for mutations in REEP1, multiplex ligation-dependent probe amplification (MLPA) suggested a heterozygous absence of exon 2 in a patient with a family history of pure, early-onset disease (Figure 1A). Long-range PCR 

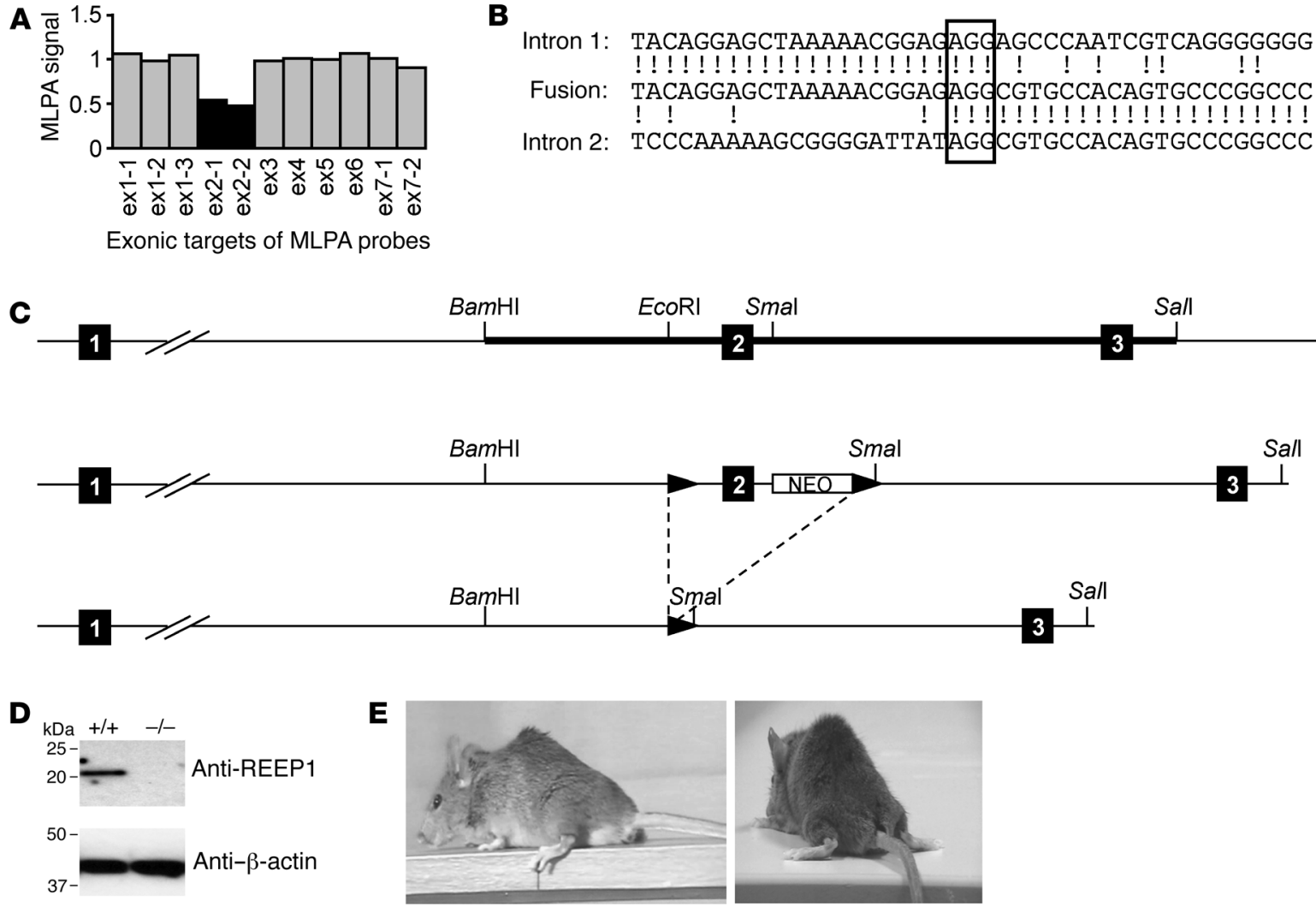

\section{Figure 1}

Lack of REEP1 exon 2 is associated with spastic paraplegia in humans and causes a severe motor phenotype in mice. (A) MLPA-based screening of genomic DNA from an HSP index patient for copy number alterations in REEP1. The assay targets exons 3-6 with single probes, and exons 1,2 , and 7 with 2 or 3 probes each (ex1-1, ex1-2, etc.). Relative MLPA signals of approximately 1.0 indicate a normal diploid copy number (gray bars), whereas signals of approximately 0.5 (black bars) indicate a heterozygous genomic deletion. (B) Alignment of REEP1 intron 1 and intron 2 sequences with the sequence of the fusion allele. The box highlights the microhomology of $3 \mathrm{bp}$ at the junction and the exclamation points denote nucleotide identity. (C) Strategy of Reep1 exon 2 deletion in mice. Wild-type locus (top schematic); the thick horizontal line marks the genomic region used for the targeting construct. The targeted locus is shown in the middle schematic, and the targeted locus after Cre-mediated exon 2 excision is shown in the bottom schematic. Numbered black squares: exons; triangles: loxP sites; NEO: neomycin selection cassette; BamHI, EcoRI, Smal, Sall: restriction sites used for cloning. (D) Western blot analysis of brain lysates from Reep $1^{+/+}$and $R e e p 1^{-/-}$mice using affinity-purified anti-REEP1 antibodies shows the absence of REEP1 protein in Reep $1^{-/-}$mice. (E) Sixteen-month-old Reep $1^{-/-}$mice suffer from gait abnormalities (see also Supplemental Videos 1 and 2). Note the broad-based positioning of the hind limbs, the kyphotic posture, and that neither the hind body nor the tail is properly lifted.

revealed that exon 2 plus flanking intronic sequences are deleted and suggested nonhomologous end-joining of adjacent double-strand breaks as the mutational mechanism (Figure 1B). The lack of exon 2 in REEP1 mRNA is predicted to create a preterminal stop codon in exon 3 (Supplemental Figure 1; supplemental material available online with this article; doi:10.1172/JCI65665DS1).

In order to study the pathophysiology of REEP1-associated HSP, we modeled the exon 2 deletion in mice (Figure 1C and Supplemental Figure 1). The effects at the protein level were examined with an antibody obtained from the immunization of rabbits against a C-terminal REEP1 epitope (Supplemental Figure 2). Whereas a band of the appropriate size was recognized in brain lysates of wild-type $\left(\right.$ Reep $\left.1^{+/+}\right)$mice, anti-REEP1 immunoreactivity was absent in mice homozygous for the Reep 1 exon 2 deletion $\left(\right.$ Reep $\left.1^{-/}\right)$. This finding confirmed the specificity of the antibody, revealed a lack of REEP1 upon exon 2 deletion, and characterized the mutation as representing a knockout allele (Figure 1D).
Mating of heterozygous mice $\left(\right.$ Reep $\left.1^{+/-}\right)$resulted in offspring of all three genotypes at Mendelian ratios. Whereas young Reep $1^{+/-}$ and Reep $1^{-/-}$mice could not be distinguished from Reep $1^{+/+}$mice by visual examination, the hind limbs of Reep $1^{-/-}$mice older than 12 months were abnormally abducted during locomotion, with extreme external rotation of the paws. Moreover, the animals frequently displayed simultaneous forward movements of both hind limbs (Supplemental Video 1). At rest, the trunk and tail were not lifted, and the paws contacted the ground with most of their plantar surface (Figure 1E) rather than showing ordinary toe placement. REEP1-deficient mice also showed spastic clonus upon a skilled walking challenge such as climbing an inclined ladder (Supplemental Video 2).

The combination of weakness and spasticity of the hind limbs, together with the apparent sparing of the forelimbs, strongly resembles the clinical symptoms of human HSP patients and thereby defines our REEP1 knockout mice as a clinically valid disease model. 
A

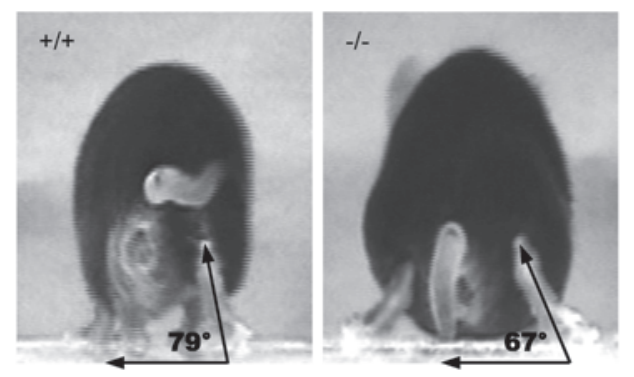

B

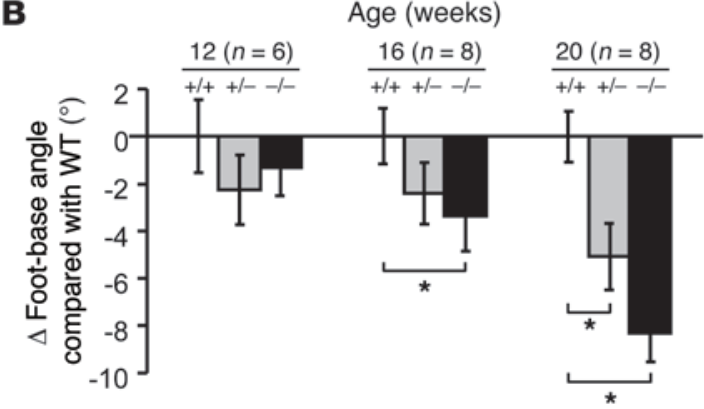

C

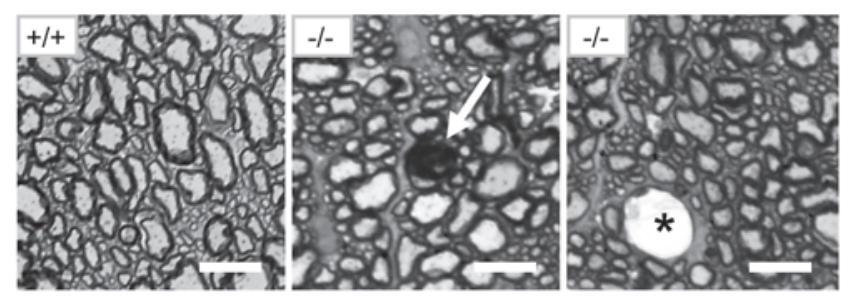

D

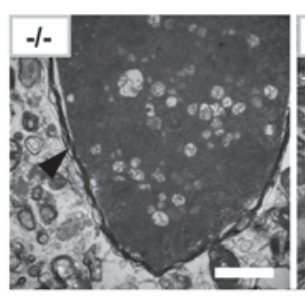

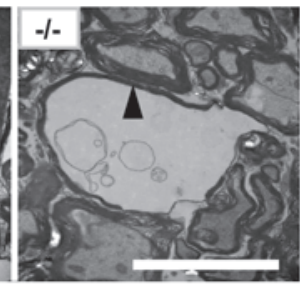

E

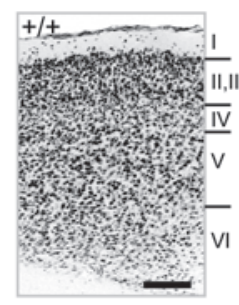

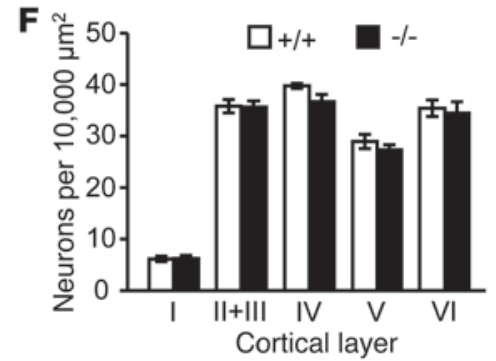

Cortical layer

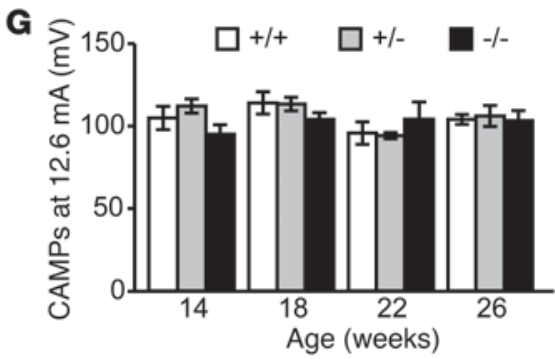

H

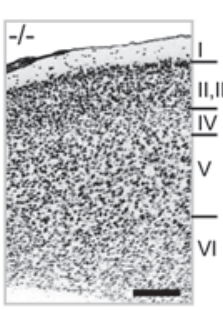

SMI
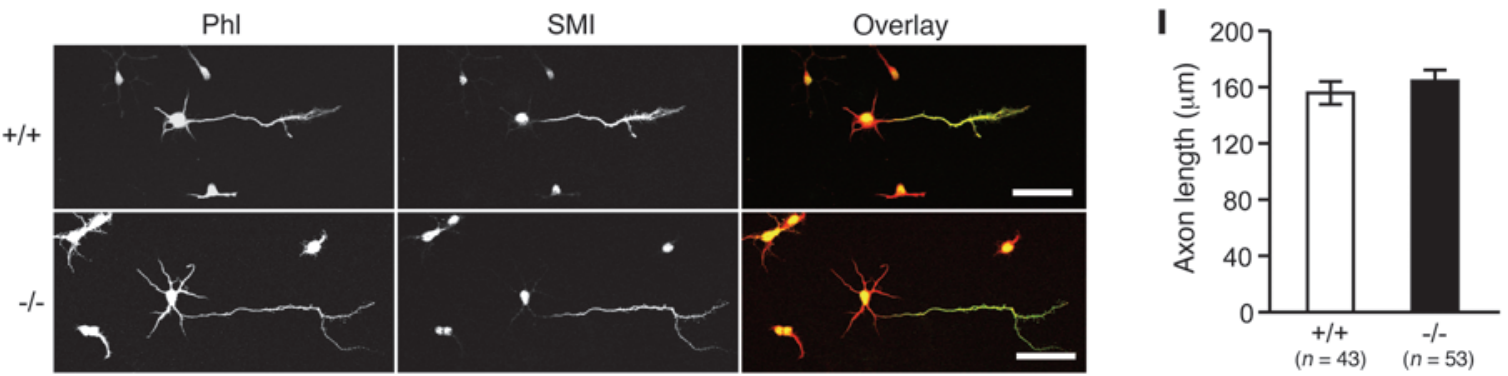

\section{Figure 2}

The REEP1 knockout phenotype is progressive, dose-dependent, and associated with a neurodegenerative rather than a neurodevelopmental pathology of upper motor neuron axons. (A) Measurement of the foot-base angle. Shown are images of 20-week-old mice immediately prior to hind paw lifting. (B) Genotype dependence of foot-base angle over time. Error bars represent the SEM. * $P<0.05$ (two-way ANOVA). (C) Semithin sections of the corticospinal tract in the spinal cord of 30-week-old mice at the lumbar level. Arrow and asterisk denote pathological structures, which are interspersed between morphological intact axons of Reep $1^{-/-}$but not Reep $p^{+/+}$mice. Scale bars: $1 \mu \mathrm{m}$. (D) Ultrastructural characterization of pathologies as highlighted in C. Dark structures are packed with electron-dense material (left panel); vacuolar structures are regions devoid of cellular material (right panel). The presence of myelin sheaths (arrowheads) indicates an axonal origin. Scale bars: $400 \mathrm{~nm}$. (E) Neuronal cell bodies in the motor cortices of 13 -month-old Reep $1^{+/+}$and Reep $1^{-/-}$mice were identified by NeuN immunoreactivity. Scale bars: $200 \mu \mathrm{m}$. (F) Layer-wise quantification revealed no neuron loss in Reep 1-l- mice. Error bars represent the SEM. (G) Lower motor neurons are not involved as judged from CMAPs upon sciatic nerve stimulation recorded from the musculus triceps surae. Error bars represent the SEM. (H) Cortical neurons obtained from P1 pups and cultured for 3 days. Neurites were visualized with phalloidin (Phl). Axons were labeled by the pan-axonal neurofilament marker SMI312 (SMI). Scale bars: $50 \mu \mathrm{m}$. (I) Quantification of the length of the longest axonal projection of each neuron. Error bars represent the SEM.

Gait impairment in REEP1-deficient mice progresses with age and is characterized by axonal degeneration in the spinal cord. We quantified the extent and progression of the movement phenotype in our mouse model by measuring the foot-base angle over time. A significant decrease was first observed in 16-week-old Reep $1^{-/-}$mice. Notably, Reep $1^{+/-}$animals were affected as well, but with later onset and to a lesser degree. At 20 weeks of age, both Reep $1^{-/-}$and Reep $1^{+/-}$mice showed strong impairments (Figure 2, A and B). 
A

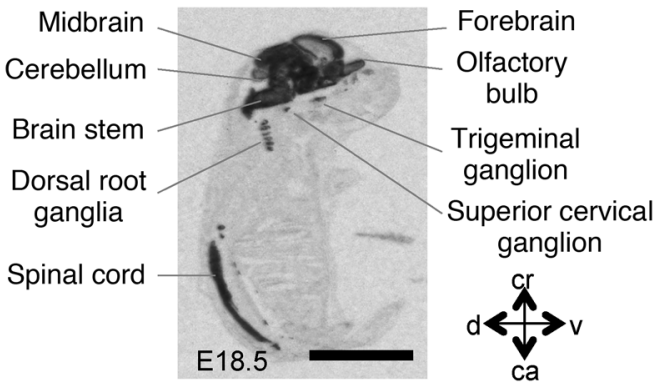

C

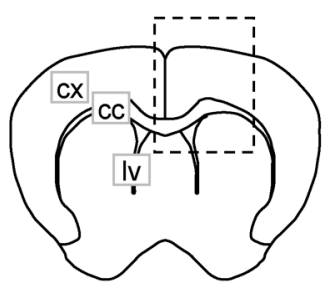

D

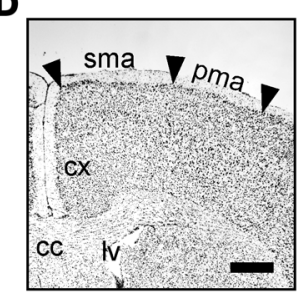

B
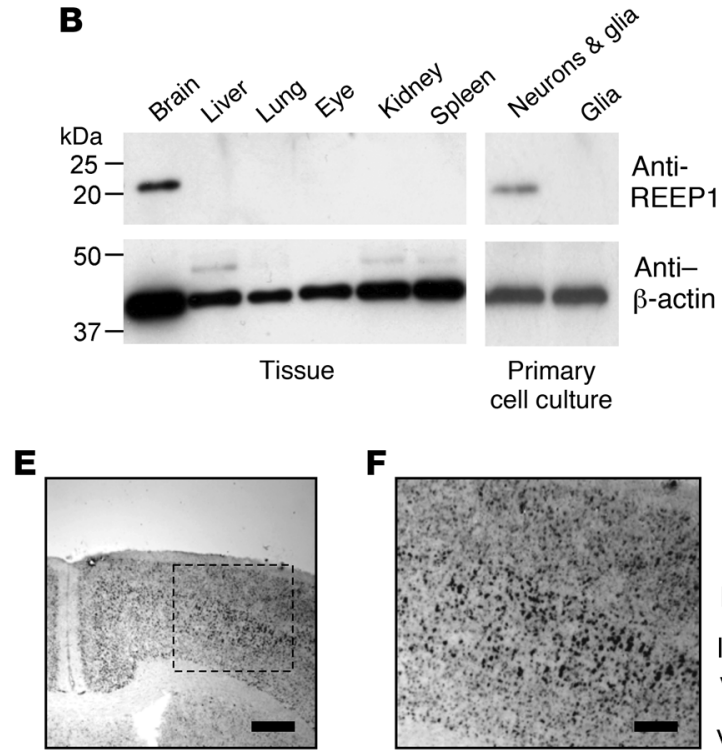

F

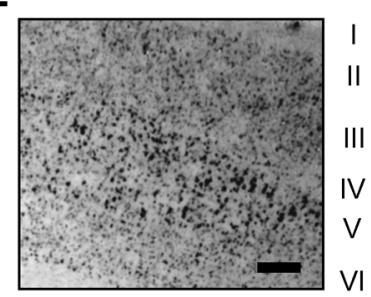

\section{Figure 3}

Reep1 expression is neuron specific and particularly strong in cortical upper motor neurons. (A) Autoradiography of in situ hybridization of an E18.5 murine sagittal embryo section using a Reep1-specific probe. ca, caudal; cr, cranial; d, dorsal; $;$, ventral. Scale bar: $3 \mathrm{~mm}$. (B) Western blot analyses of lysates of several organs from 4-month-old wild-type mice show REEP1 expression in the brain. Lysates from primary cortical cultures (P1) reveal expression in neurons but not in glial cells. (C) Schematic of a coronal section of an adult mouse brain at bregma $+0.7 \mathrm{~mm}$. cc, corpus callosum; cx, cortex; Iv, lateral ventricle. Box depicts area displayed in D and E. (D) Nissl staining of a section of a 4-month-old wild-type mouse. pma, primary motor area; sma, secondary motor area. Arrowheads show the area borders. Scale bars: $500 \mu \mathrm{m}$. (E) In situ hybridization with a Reep1-specific probe on the section adjacent to the section shown in D. Scale bar: $500 \mu \mathrm{m}$. (F) Magnification of the primary motor area. Note the particularly strong labeling of cells in layer V of the primary motor cortex (i.e., upper motor neurons). Scale bar: $200 \mu \mathrm{m}$.

Cross sections of the spinal cord at lumbar levels in 30-week-old Reep $1^{-/-}$mice revealed evidence of corticospinal tract axon degeneration (Figure 2, C and D). There was, however, no loss of cortical motor neuron somata even at 13 months of age (Figure 2, E and F).

Considering the lower motor neuron involvement in the SPG7 mouse model $(19,20)$ and in patients carrying a toxic gain-offunction REEP1 allele (21), we measured compound muscle action potentials (CMAPs) in the musculus triceps surae, as alterations in this parameter have been correlated with preclinical loss of spinal cord motor neuron axons in a mouse model for amyotrophic lateral sclerosis (22). Supporting the finding that lower motor neurons were not affected, CMAPs in Reep ${ }^{+/-}$and Reep $1^{-/-}$animals did not differ from those in Reep $1^{+/+}$controls even 10 weeks after the onset of gait impairment (Figure 2G).

In order to address whether axon outgrowth is impaired in the absence of REEP1, we isolated cortical neurons from P1 mice and spinal cord motor neurons from E12.5 embryos and quantified the axon length after 3 (cortical neurons) or 5 (spinal cord motor neurons) days in culture, respectively. There was no evidence of shorter axons or abnormal axon morphologies in neurons isolated from Reep $1^{-/-}$mice (Figure 2, H and I, and Supplemental Figure 3).

Based on the adult onset of the phenotype, its progressive and degenerative rather than developmental nature, and the restriction of the defect to upper motor neuron axons, we conclude that the REEP1-deficient mouse is a valid model to study the pathophysiology behind axon loss in SPG31.

Reep1 expression is neuron specific and particularly prominent in upper motor neurons. Radioactive whole-mount in situ hybridization on sagittal mouse sections revealed that Reep 1 transcripts are already expressed during embryonic development and are confined to the nervous system (Figure 3A and Supplemental Figure 4).

Consistent with these findings, REEP1 protein was detected in brain lysates but not in lysates of any of several other tissues analyzed (Figure 3B, left panel). Moreover, a REEP1-derived signal was present in lysates of mixed neuronal glial cell cultures, but was absent from lysates of pure glial cell cultures (Figure 3B, right panel), indicating that REEP1 is specific to neurons.

Since our antibody did not detect endogenous REEP1 in immunofluorescence studies, we performed nonradioactive in situ hybridizations in order to define Reep 1 expression in the brain at the cellular level. The particularly strong labeling of cells in layer $\mathrm{V}$ of the primary motor cortex in coronal brain sections suggests a predominant expression of Reep1 in upper motor neurons (Figure 3, C-F).

The phenotype and pathology of the REEP1 knockout as defined above are therefore consistent with both the tissue and cell specificity of Reep 1 expression.

REEP1 associates with cellular membranes and is enriched in the ER. REEP1 contains two hydrophobic domains that have been proposed to mediate membrane association (23). Its intracellular localization, however, has been controversial. Reports have included plasma membrane (23), mitochondria (8), or ER (11) localization of REEP1. In protein lysates prepared from mouse brain, we detected REEP1 exclusively in a crude membrane fraction, but not in the cytosolic or mitochondrial fractions (Figure 4A). In subfractions of the postmitochondrial fraction, REEP1 detection overlapped with the tubular ER marker reticulon 4 (RTN4) (16) rather than with the trans-Golgi marker $\gamma$-adaptin (ref. 24 and Figure 4, B and C). 

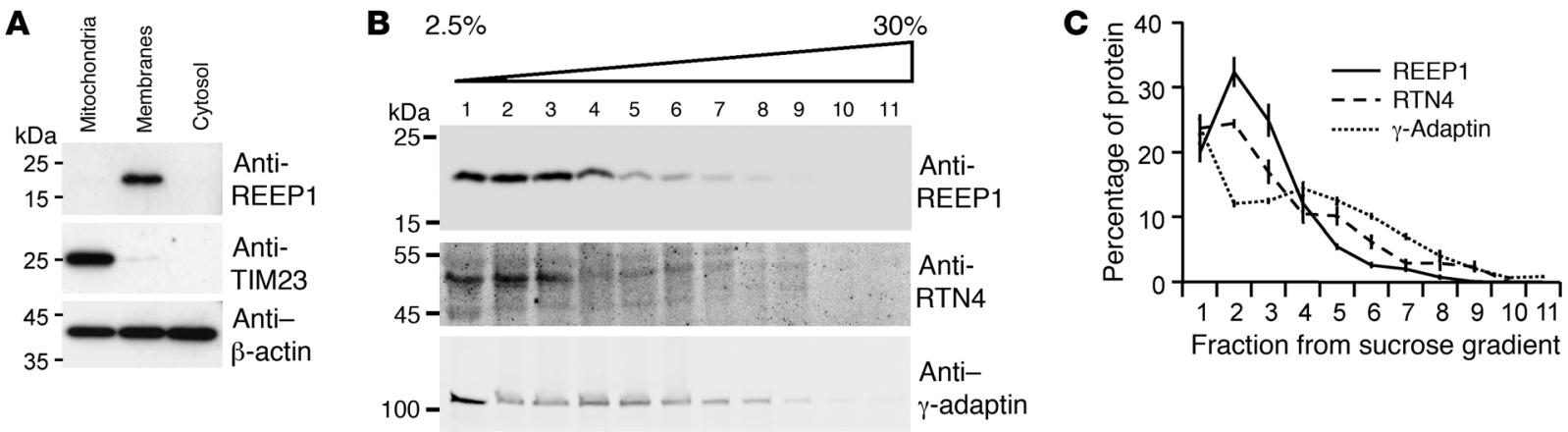

\section{Figure 4}

REEP1 is enriched in cellular membranes that contain the ER protein RTN4. (A) Immunoblotting of subcellular fractions of murine brain lysates. TIM23: mitochondrial marker; actin served as a loading control. (B) Western blot analyses of REEP1, RTN4, and $\gamma$-adaptin after further subfractionation of the REEP1-containing postmitochondrial fractions using iodixanol-gradient centrifugation. (C) Quantification of the data shown in B $(n=4)$. REEP1 cofractionates with the ER marker RTN4 but not with $\gamma$-adaptin, a marker for the trans-Golgi. Error bars represent the SEM.

These observations suggest a localization of REEP1 to the ER and particularly to highly curved ER compartments.

Liposome binding by REEP1 increases membrane curvature in vitro. As the central hydrophobic domain of REEP1 is unusually long and may serve as a hydrophobic wedge that is inserted into one leaflet of lipid bilayers to induce positive membrane curvature, we incubated liposomes with purified REEP1. We used recombinant N-terminally HisTrx-tagged REEP1 (HisTrx-REEP1) and a mutant, in which the hydrophobic residues I57, F58, F62, and F64 in the REEP domain were each exchanged by the polar amino acid serine (HisTrx-REEP1-mut). Both proteins, but not the HisTrx control, floated with liposomes into fraction 2 of density-gradient centrifugations (Figure 5, A and B).

In order to reveal the putative membrane-shaping activity of REEP1, snap-frozen protein-liposome suspensions were freeze-fractured, and the liposome size was determined by transmission electron microscopy (TEM). The diameters of liposomes incubated with tagged or N-terminally untagged REEP1 were drastically reduced compared with the HisTrx control (Figure 5, C-E, and Supplemental Figure 5). Specifically, a group of liposomes with diameters of only $30 \pm 10 \mathrm{~nm}$ that was virtually absent in the control incubations was very prominent upon incubation with REEP1 (Figure 5D).

This size-reduction effect was strongly diminished in incubations with HisTrx-REEP1-mut (Figure 5, C-E), indicating that the hydrophobic residues I57, F58, F62, and F64 of the REEP domain are critical in promoting membrane curvature. Further supporting its role in active membrane shaping, REEP1 was preferentially localized at the surface of small-diameter liposomes (Figure 5F). Moreover, live imaging of protein-liposome suspensions clearly showed that liposome size remained stable over time upon the addition of the HisTrx control, whereas the addition of HisTrx-REEP1 rapidly induced constrictions and conversion of large liposomes into smaller ones (Figure 5G and Supplemental Videos 3 and 4). Consistent data were obtained with N-terminally untagged REEP1 (Supplemental Figure 5E and Supplemental Videos 5 and 6).

These observations suggest that REEP1 association with membranes promotes their positive curvature, and that this REEP1 function requires the integrity of the REEP domain.

REEP1 knockout mice show a dose-dependent reduction in the complexity of the peripheral ER in cortical motor neurons. Considering (a) the phenotypical consequences of REEP1 deficiency; (b) the neu- ron-specific expression of the gene; (c) the ER association of the protein; and (d) its membrane-shaping properties, we hypothesized that REEP1 may play a critical role in proper organization of the neuronal ER, which consists of the nuclear envelope and its peripheral components. Both subcompartments were evaluated in ultrastructural sections of layer $V$ neurons of the primary motor cortex (Figure 6A).

Regarding the nucleus, neither the total length of the nuclear membrane (Figure 6B), the sphericity of the nuclear section (Figure 6C), nor the number of nuclear pockets per section (Figure 6D) showed any differences between genotypes.

Likewise, there was no difference in the cumulative length of peripheral ER structures (Figure 6E). The length of the individual structures, however, showed a statistically significant increase in Reep $1^{-/-}$mice (Figure 6F). This gain in length was paralleled by statistically significant reductions in the number of peripheral ER structures in both Reep $1^{+/-}$and Reep ${ }^{-/-}$mice (Figure 6G).

These findings at the ultrastructural level imply that REEP1 deficiency has no effect on the nuclear portion of the ER. Instead, the observation of fewer but longer structures in the peripheral ER suggests that the complexity of this second subcompartment negatively correlates with the amount of REEP1 available in the neuron.

\section{Discussion}

The aim to better characterize the factors that are important for axonal maintenance prompted us to model SPG31 in mice. Based on our identification of a deletion of exon 2 in REEP 1 in a patient suffering from HSP, we removed the corresponding exon 2 in mice by a Cre-lox strategy. The deletion causes a frame shift resulting in a Reep1 knockout allele, which was confirmed by the absence of REEP1 protein in brain lysates of homozygously targeted mice. During early adulthood, Reep $1^{+/-}$mice developed a progressive movement disorder of the hind limbs with weakness, spasticity, and degeneration of axons of the corticospinal tract mirroring the human SPG31 phenotype. Homozygous Reep $1^{-/-}$mice became symptomatic 4 weeks earlier and were more severely affected, but the restriction of the pathology to upper motor neurons was preserved. The correlation between the phenotype and the number of knockout alleles establishes loss of function as the leading pathomechanism in REEP1-related HSP and suggests that HSP in patients with other REEP1 mutations also reflect loss of func- 
A

HisTrx
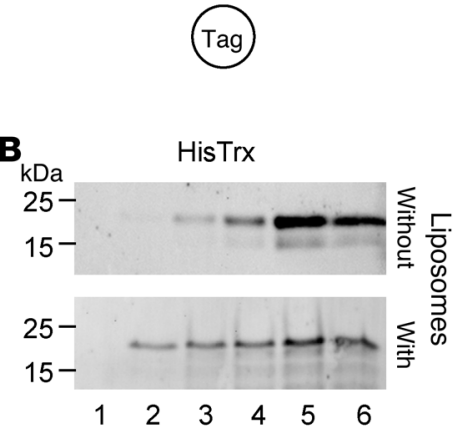

HisTrx-REEP1

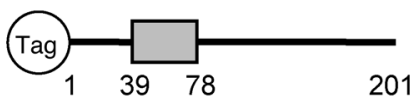

HisTrx-REEP1-mut

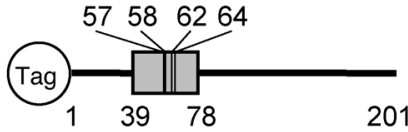

C

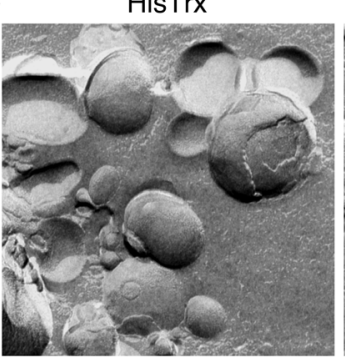

HisTrx-REEP1

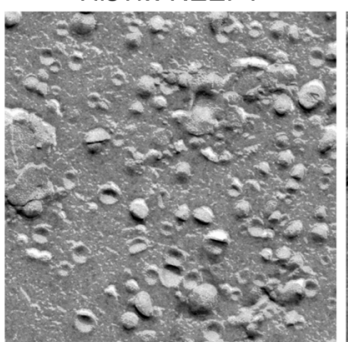

HisTrx-REEP1-mut

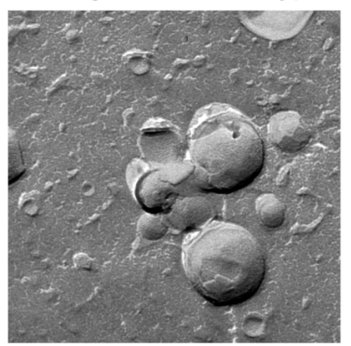

HisTrx-REEP1-mut

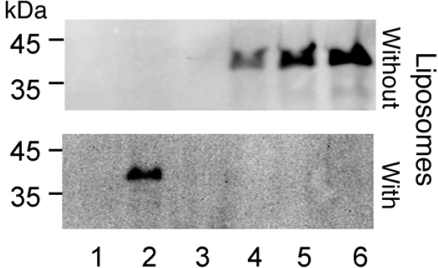

D

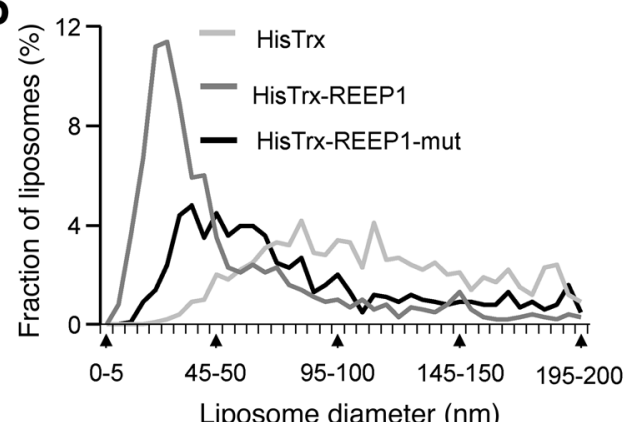

E

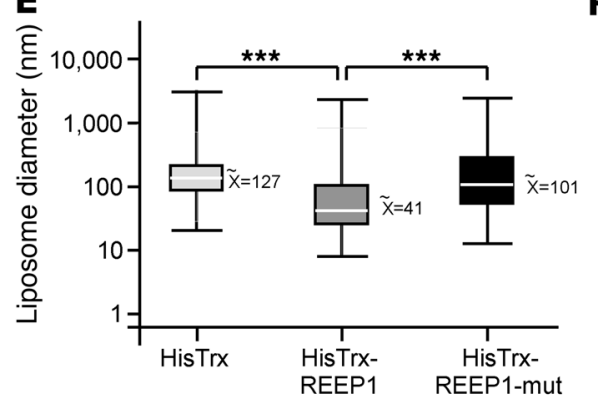

F

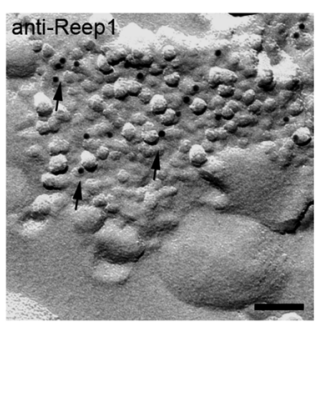

G

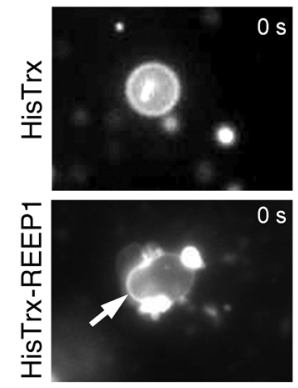

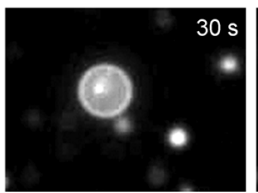

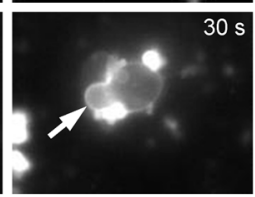

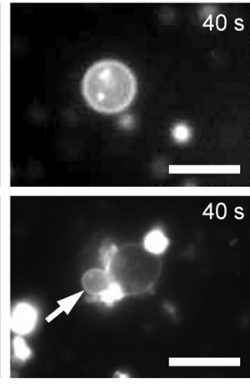

Figure 5

REEP1 binds and curves membranes in vitro. (A) Constructs used for liposome-binding assays. The hydrophobic region encompassing residues 39-78 of REEP1 is marked (gray box). The positions of hydrophobic residues mutated to serines in HisTrx-REEP1-mut are indicated. (B) Liposome cofloatation assays. Proteins were detected using anti-HisTrx antibodies in immunoblots of sucrose gradient fractions 1 (top) to 6 (bottom). Tagged REEP1 and REEP1-mut, but not the tag alone, floated with liposomes in fraction 2. (C) TEM images of freeze-fractured incubations of liposomes with the indicated recombinant proteins. Scale bars: $200 \mathrm{~nm}$. (D) Distribution of liposome diameters observed by TEM of freeze-fractured liposome incubations. Note that incubation with REEP1 leads to a pronounced increase in the relative numbers of 20- to 40-nm structures, the frequencies of which are strongly diminished upon incubation with HisTrx-REEP1-mut and are largely absent in the control incubations. (E) Box plots of the full set of data partially presented in C. Note that the $y$ axis is logarithmic. ${ }^{* * *} P<0.001$ (one-way ANOVA). Boxes contain $50 \%$ of the values; minimal, maximal, and median values are marked by vertical lines. (F) High-resolution TEM analysis of freeze-fractured liposomes incubated with REEP1 and immunogold labeled for REEP1 shows REEP1 at positively curved membranes of predominantly small liposomes (arrows). Scale bar: $80 \mathrm{~nm}$. (G) Sequential video frames of liposomes incubated with HisTrx control protein and HisTrx-REEP1 (taken from Supplemental Videos 3 and 4). Arrows indicate the constriction of a large liposome into two smaller, stronger curved membrane structures upon addition of HisTrx-REEP1. Scale bars: $5 \mu \mathrm{m}$.

tion and not dominant-negative effects of truncated or mutated REEP1 protein variants.

There have been conflicting data regarding the sites of Reep1 expression. While some have reported ubiquitous expression (8), others exclusively detected Reep 1 in the brain (23). Here, we demonstrate that Reep1, though expressed throughout the nervous system, shows particularly strong expression in cell bodies of upper motor neurons in cortical layer V. This is consistent with the main pathology seen in pure HSP. From our Western blot analysis of cultured neurons and glial cells, we further conclude that the REEP1 protein is neuron specific. In contrast, SPAST, which is mutated in SPG4 and is associated with a similar phenotype and pathology to that of REEP1, has a much broader expression (25). Regarding the developmental pattern of Reep1 expression, its tran- 
A
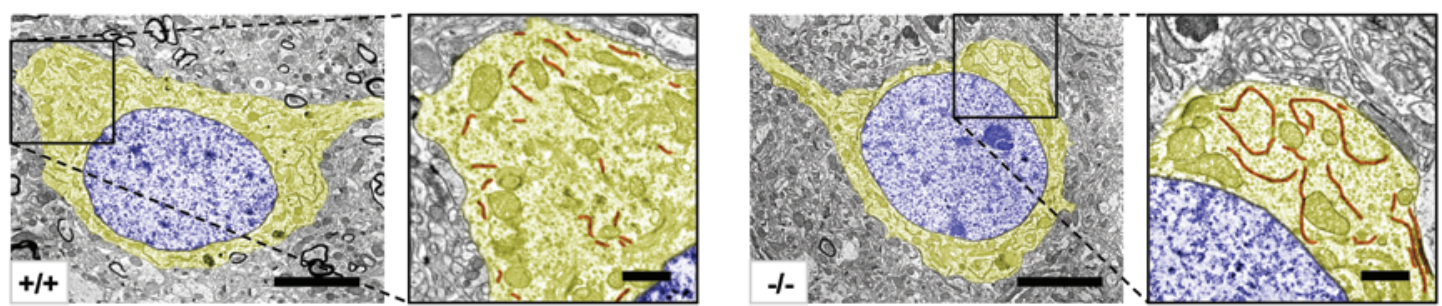

B

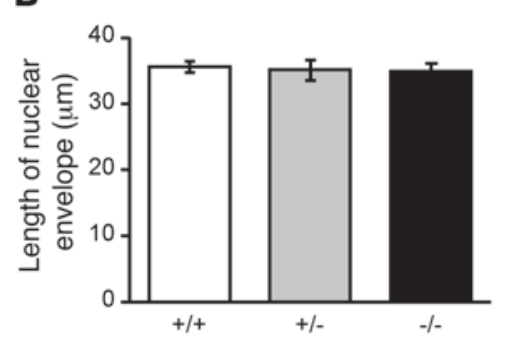

E

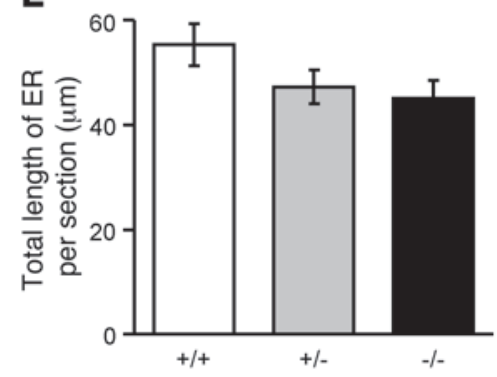

C

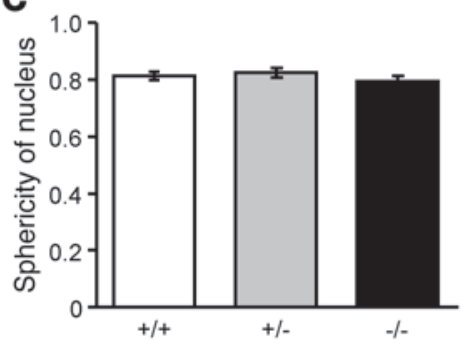

F

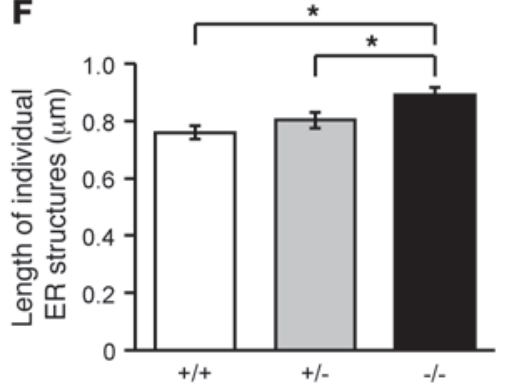

D
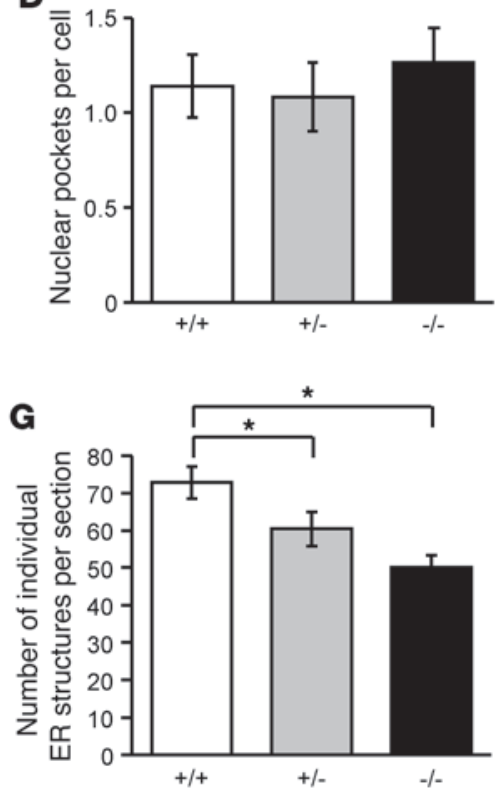

Figure 6

REEP1 deficiency results in a dose-dependent reduction of the complexity of the peripheral ER but not of the nuclear envelope in neurons in the primary motor cortex. (A) Representative TEM images of neuronal somata in the primary motor cortex of Reep ${ }^{+/+}$and Reep $1^{-/-}$mice. The cytoplasm is colored in yellow, the nucleus in blue, and the ER in red. Scale bars: $2 \mu \mathrm{m}$ for whole-cell images and $400 \mathrm{~nm}$ for insets. (B-G) Quantitative evaluations (mean \pm SEM) of nuclear envelope and peripheral ER parameters in TEM sections of neuronal somata in the primary motor cortex of Reep $1^{+/+}, R e e p 1^{+/}$, and Reep1 $1^{-/}$mice (4 animals per genotype, 12 cells per animal). Neither the length of the nuclear envelope (B), its sphericity $(\mathbf{C})$, nor the number of nuclear pockets observed per cell section (D) differed between genotypes. Although the sum of the length of all peripheral ER structures per cell section was unaltered (E), a REEP1 dose-dependent increase was observed in the average length of individual ER structures (F) associated with a decrease in the number of individual ER structures per cell section (G). ${ }^{*} P<0.05$ (one-way ANOVA).

scripts were already abundant in the nervous system at embryonic stages, however, Reep $1^{+/-}$and Reep $1^{-/-}$mice developed normally and had neither motor deficits nor gross alterations of the brain or spinal cord axons in their first months of life. Moreover, axonal outgrowth in neurons cultured from REEP1-deficient mice was unaffected. Thus, REEP1 is most likely not crucial for brain and spinal cord development, which fits well with the lack of phenotypes of REEP1 mutations in humans during early childhood and is consistent with the view that HSP generally manifests as a neurodegenerative rather than a neurodevelopmental disease (26).

Regarding the subcellular distribution of REEP1, conflicting reports have been published, which can be attributed to difficulties in the detection of endogenous REEP1. In agreement with overexpression studies in HEK293 and HeLa cells (11), our Western blot analysis of subcellular fractions of brain lysates clearly shows that endogenous REEP1 is associated with ER membranes, whereas the mitochondria, where REEP1 was initially reported to localize (8), were devoid of REEP1.
Our in vitro reconstitution assays show that REEP1 directly associates with lipids. Since we used liposomes, i.e., reconstituted membranes, it can further be concluded that REEP1 is not only able to bind to lipid molecules, but can also very effectively associate with lipid bilayers. Since our assay was devoid of any other proteins, it can furthermore be concluded that this high affinity for lipid bilayers does not reflect a signal-dependent transmembrane integration, but rather is inherent to REEP1.

The live microscopic analyses and quantitative examinations of in vitro reconstitutions further show that association of REEP1 with membranes promotes positive membrane curvature, causing the constriction of liposomes and a corresponding substantial increase in the abundance of small-diameter liposome structures. In line with these data, REEP1 immunogold labeling was restricted to the surface of smaller liposomes. So far, curvature-promoting properties of ER proteins have been experimentally shown in reticulons and REEP5/Yop1p $(14,16)$, which are characterized by the presence of paired hydrophobic domains with unusual lengths 
thought to both insert into highly curved membranes as wedges. REEP1 is set apart from the reticulons and REEP5/Yop1p because of its shorter N-terminal hydrophobic domain $(11,12,27)$. Our data show that the second, longer hydrophobic domain is sufficient and its integrity critical for the shaping activity of REEP1.

In order to gain insights for the first time into the pathophysiology of REEP1 deficiency at the subcellular level in vivo, we conducted a detailed morphometric analysis of the ER in layer $\mathrm{V}$ neurons of the motor cortex, i.e., a region in which wild-type mice show particularly strong Reep 1 expression. Quantitative evaluations showed that the number of ER structures was decreased in both Reep $1^{+/-}$and Reep $1^{-/-}$mice, whereas the length of individual ER compartments was increased. Reduced ER complexity corresponds to a reduced abundance of sharply curved ER membrane surfaces such as those found at the rims of ER sheets, at fenestrae, and at tubules. These dynamic ER substructures have a positive curvature with a relatively uniform diameter of about 35 to 40 $\mathrm{nm}$ (28). This value strikingly mirrors our in vitro finding that liposomes with diameters of about $40 \mathrm{~nm}$ are the most abundant in the presence of REEP1. Supporting this, RNAi-based knockdown of the Drosophila reticulon Rtnl1 in epidermal cell cultures was recently reported to increase the size of ER structures (29). As reticulons have also been suggested to bend membranes, these observations may correspond to reduced ER membrane curvature similar to the effects we observed upon REEP1 knockout.

Layer $\mathrm{V}$ motor neurons project over an extremely long distance onto spinal cord motor neurons, thus it is conceivable that these neurons depend on a particularly elaborate ER to maintain their long axonal extensions. This is supported by evidence that proteins implicated in three other major types of dominant HSPs are also linked to the ER: ATL1, mutated in SPG3A, encodes ATLASTIN1, which was shown to directly interact with tubule-shaping proteins to promote homotypic fusion of ER membranes and maintain proper ER network formation $(30,31)$. SPAST, mutated in SPG4, encodes SPASTIN, a protein that was shown to interact with ATLASTIN1 and REEP1 and organizes the structure of the ER by connecting it to microtubules (11). Finally, RTN2, which is mutated in SPG12 (32), encodes a protein of the reticulon family that has been shown to reside in and shape the ER (29).

Thus, REEP1 knockout in mice and comparison with the clinical, neurodegenerative phenotype of the autosomal dominantly inherited HSP variant SPG31 revealed that loss of REEP1 leads to defects in ER organization. Whether an altered ER complexity may also interfere with ER functions like the unfolded protein response and/or specific ER export functions are important questions to address in the future.

\section{Methods}

REEP1 copy number screening in HSP patients. Eighty-seven index patients from Spanish families with dominant HSP were enrolled (33). MLPA was performed according to the manufacturer's instructions using the SALSA P213 probe mix (MRC-Holland). The sample with the aberrant signals for exon 2 was subjected to long-range PCR, applying a forward primer in intron 1 and a reverse primer in intron 2 , and was subsequently sequenced (Supplemental Table 1).

Modeling Reep1 exon 2 deletion in mice. An 8.3-kb fragment of a 129/SvJ mouse genomic $\lambda$ library (Stratagene) including exons 2 and 3 and neighboring intronic sequences was cloned into the pKO-V901 plasmid (Lexicon Pharmaceuticals) containing a phosphoglycerate kinase (PKG) promoter-driven diphtheria toxin A cassette using Bam HI and SalI sites. One
loxP site was inserted approximately $1.2 \mathrm{~kb}$ upstream of exon 2, thereby destroying an EcoRI site. A PGK promoter-driven neomycin resistance cassette flanked by loxP sites and preceded by an EcoRI site was inserted into an SmaI site 262 bp downstream of exon 2 . The construct was electroporated into R1 mouse ES cells. Neomycin-resistant clones were analyzed by Southern blotting of EcoRI-digested ES cell DNA. Correctly targeted ES cells were transfected with a plasmid expressing Cre recombinase. Recombination events removing exon 2 as well as the neomycin resistance cassette were identified upon Southern blotting of EcoRI-digested genomic DNA. One of the positive clones was injected into C57BL6 blastocysts. The resulting chimeras were subsequently backcrossed with C57BL6 to obtain Reep $1^{+/-}$mice. Studies were performed with littermates of the F4 generation. Genotypes were determined by PCR on DNA isolated from tail biopsies (Supplemental Table 1).

Antibodies. Anti-REEP1 antisera were raised by immunization of rabbits against the peptide GRSSGKHSQPKMSRS (residues 176-190) (Eurogentech) and affinity purified as described (34). Other primary antibodies used were: rabbit anti- $\beta$-actin (Abcam); mouse anti-TIM23 (BD Biosciences); rabbit anti-TUBB3 ( $\beta 3$-tubulin; Sigma-Aldrich); mouse anti- $\gamma$-adaptin (BD Transductions); rabbit anti-reticulon-4 (Aviva Systems Biology); mouse antiSMI312 (Hiss Diagnostics); mouse anti-NeuN (Millipore); rabbit anti-phospho-tau (Biozol Diagnsotika); mouse anti-MAP2ab (Acris Antibodies); and rabbit anti-HisTrx antibodies raised and affinity purified as described (35).

Gait analysis. The foot-base angle at toe-off positions of the hind paws was quantified using single video frames from recordings of beam-walking mice (36). Statistical analyses were performed using two-way ANOVA. A skilled walking challenge was imposed by an inclined ladder (rung distance: $2 \mathrm{~cm}$; rung thickness: $2 \mathrm{~mm}$; rung width: $6 \mathrm{~cm}$; inclination: $\left.45^{\circ}\right)(37)$ and evaluated qualitatively.

Histological analysis of spinal cord and brain. For immunohistochemical analysis of the motor cortex in 13-month-old Reep $1^{+/+}$and Reep P $^{-/}$animals, neuronal cell bodies were visualized by NeuN staining of $20-\mu \mathrm{m}$ sagittal cryosections ( $n=6$ per genotype) and quantified layerwise. For semiand ultrathin sectioning, animals were perfused with $50 \mathrm{ml}$ fixative (4\% paraformaldehyde, $1 \%$ glutaraldehyde). The brain and spinal cord were removed and postfixed overnight. Coronal sections $(150 \mu \mathrm{m})$ were cut with a vibratome (Leica Microsystems) and processed as described (38). Semithin sections were stained with methylene blue. Ultrathin 80 -nm sections (Ultratome III; LKB Instruments) were mounted on filmed copper grids (100 mesh), poststained with lead citrate, and studied using a TEM (EM 900; Zeiss) at $80 \mathrm{kV}$.

Quantitative analysis of electron microscopy images. For morphometry, only sections of somata of pyramidal neurons not extending beyond the image boundary and hit vertically in respect to the nuclear envelope were selected. Files were uploaded using ImageJ software (NIH), and the cell boundary, the nucleus, and the complete set of sheet-like ER structures were traced for each cell with a touch-sensitive pen screen system. The sphericity of the nucleus was calculated as the area of the nucleus divided by the area of a circle within the given perimeter. Data were obtained for four 12-monthold animals per genotype and 12 neurons per animal. Statistical analyses were performed using one-way ANOVA.

Electromyographical analysis. Evoked CMAP amplitudes were evaluated as described (22). Statistical analyses were performed using two-way ANOVA. In situ bybridization. As a probe template, a murine Reep1 cDNA fragment was amplified and cloned into PCRII-TOPO (Invitrogen). Radioactive (39) and nonradioactive (21) in situ hybridizations were carried out as described.

Analysis of axon outgrowth and ER stress in neuron cultures. Axon length in 5-day-old spinal cord motor neuron cultures from E12.5 embryos was determined as reported previously (40). Cortical neurons and glia cells were prepared from P0 or P1 animals and cultured as described (41). Cells were 
fixed after 3 days in culture and stained for F-actin and immunostained with antibodies against the pan-axonal neurofilament marker SMI312. Images of neurons were acquired at $\times 40$ magnification (Axioplan2; Zeiss), and axon length was measured using ImageJ software. Statistical analyses were performed using the Student's $t$ test.

Subcellular fractionation. Four wild-type mice were sacrificed and tissues immediately removed and snap-frozen at $-80^{\circ} \mathrm{C}$. Tissues were ground using a precooled mortar and pestle and homogenized using a glassTeflon device (Braun Biotech) as described (34). Subcellular fractions were obtained by differential centrifugation at $4^{\circ} \mathrm{C}(10$ minutes at $1,000 \mathrm{~g}$ to pellet nuclei, 15 minutes at $10,000 \mathrm{~g}$ to pellet mitochondria, 1 hour at $100,000 \mathrm{~g}$ to pellet the membrane fraction). The postmitochondrial fraction was further fractionated by a discontinuous iodixanol gradient, and individual fractions were diluted, pelleted, resolved in SDS sample buffer (42), and quantitatively evaluated by fluorescence-based immunoblotting using a LI-COR Odyssey system.

Purification of recombinant REEP1. The coding sequence of human REEP1, including the stop codon, was amplified from brain-derived cDNA (Clontech) and cloned into the pET32-a vector (Novagen). From this construct (HisTrx-REEP1), the construct for the HisTrx-REEP1-mut variant was generated by PCR mutagenesis-mediated introduction of four single base alterations (p.I57S, p.F58S, p.F62S, and p.F64S). His-tagged fusion proteins were purified from $E$. coli as described (35).

Liposome cofloatation assay. Liposomes were prepared as described (43). Liposomes $(150 \mu \mathrm{g})$ were incubated with $0.5 \mu \mathrm{M}$ protein for 15 minutes at $37^{\circ} \mathrm{C}$ in $0.3 \mathrm{M}$ sucrose in liposome buffer ( $25 \mathrm{mM}$ HEPES-KOH, pH 7.2; $25 \mathrm{mM} \mathrm{KCl} ; 2.5 \mathrm{mM} \mathrm{Mg}$-acetate; $100 \mathrm{mM} \mathrm{K}$-glutamate) and mixed with $150 \mu \mathrm{l}$ $75 \%$ sucrose in liposome buffer. The mixture was overlaid with $200 \mu 135 \%$ sucrose and $200 \mu \mathrm{l}$ liposome buffer and then centrifuged at $200,000 \mathrm{~g}$ for 30 minutes at $28^{\circ} \mathrm{C}$. Six fractions containing $100 \mu \mathrm{l}$ eluate each were collected from top to bottom and analyzed by SDS-PAGE and immunoblotting.

Live imaging of liposome-protein mixtures. Liposomes were supplemented with $5 \%$ rhodamine PE (Avanti Polar Lipids), incubated with $0.1 \mu \mathrm{M}$ of the recombinant proteins at room temperature for 10 minutes, and visualized (Zeiss Observer Z1; Zeiss). Images were acquired every 200 milliseconds over a period of 2 minutes.

Liposome-shaping assay. For freeze-fracture experiments, $1 \mathrm{mg}$ of liposomes was incubated with $5 \mu \mathrm{M}$ protein in liposome buffer containing $0.3 \mathrm{M}$ sucrose for 15 minutes at $37^{\circ} \mathrm{C}$. To avoid liposomal aggregates, $20 \mu \mathrm{g}$ proteinase $\mathrm{K}$ was subsequently added, followed by another incubation at $45^{\circ} \mathrm{C}$ for 40 minutes. Small aliquots $(1-2 \mu \mathrm{l})$ of vesicle suspension were freeze-fractured as described (41). Immunogold labeling at freeze-fracture replicas without proteinase $\mathrm{K}$ incubation was done using specific antiREEP1 primary antibodies (1:50) followed by a gold-conjugated secondary antibody (1:50, $10 \mathrm{~nm}$ gold; British Biocell International). The samples were examined using an EM 902 A electron microscope (Zeiss). Images were acquired using a $1 \mathrm{k}$ FastScan CCD camera (TVIPS). Diameters of 900-1,300 liposome structures per incubation condition were determined using ImageJ software.

Statistics. One-way ANOVA was used for comparisons between three groups (for liposome diameters and for the ultrastructural analysis of ER structures). A two-tailed Student's $t$ test was used for comparison between two groups (axon outgrowth and quantification of cortical neurons). For the analysis of repeated measurements over time, two-way ANOVA was applied (gait analysis, CMAP analysis). $P \leq 0.05$ was considered statistically significant.

Study approval. All animal experiments were approved by the local administrative institution (Thüringer Landesamt für Verbraucherschutz, Bad Langensalza, Germany). Written informed consent was obtained from the patients for genetic studies.

\section{Acknowledgments}

We thank M. Öhler, B. Schade, K. Schorr, A. Büschel, K. Stein, and R. Kaiser for their excellent technical assistance. We thank C. Kaether for valuable discussions and Laura McMillan for editing the final manuscript. This work was supported by grants from the Deutsche Forschungsgemeinschaft (DFG) to M.M. Kessels (KE685/3-1), B. Qualmann (QU116/6-1), C. Beetz (BE4069/1-1), T. Deufel and C.A. Hübner (DE307/8-1), and C.A. Hübner (HU800/6-1).

Received for publication July 6, 2012, and accepted in revised form July 3, 2013.

Address correspondence to: Christian A. Hübner, Institute of Human Genetics, Jena University Hospital, D-07743 Jena, Germany. Phone: 0049.3641.935500; Fax: 0049.3641.935502; E-mail: christian.huebner@med.uni-jena.de.
1. Goldberg JL. How does an axon grow? Genes Dev. 2003;17(8):941-958.

2. Zuchner S, Vance JM. Emerging pathways for hereditary axonopathies. J Mol Med. 2005;83(12):935-943.

3. Harding AE. Hereditary spastic paraplegias. Semin Neurol. 1993;13(4):333-336

4. Schule R, Schols L. Genetics of hereditary spastic paraplegias. Semin Neurol. 2011;31(5):484-493.

5. Blackstone C. Cellular pathways of hereditary spastic paraplegia. Annu Rev Neurosci. 2012;35:25-47.

6. Salinas S, Proukakis C, Crosby A, Warner TT. Hereditary spastic paraplegia: clinical features and pathogenetic mechanisms. Lancet Neurol. 2008; 7(12):1127-1138

7. Blackstone C, O'Kane CJ, Reid E. Hereditary spastic paraplegias: membrane traffic and the motor pathway. Nat Rev Neurosci. 2011;12(1):31-42.

8. Zuchner S, et al. Mutations in the novel mitochondrial protein REEP1 cause hereditary spastic paraplegia type 31. Am J Hum Genet. 2006;79(2):365-369.

9. Beetz C, et al. REEP1 mutation spectrum and genotype/phenotype correlation in hereditary spastic paraplegia type 31. Brain. 2008;131(4):1078-1086.

10. Goizet C, et al. REEP1 mutations in SPG31: frequency, mutational spectrum, and potential association with mitochondrial morpho-functional dysfunction. Hum Mutat. 2011;32(10):1118-1127.
11. Park SH, Zhu PP, Parker RL, Blackstone C. Hereditary spastic paraplegia proteins REEP1, spastin, and atlastin- 1 coordinate microtubule interactions with the tubular ER network. J Clin Invest. 2010; 120(4):1097-1110.

12. Bauer M, Pelkmans L. A new paradigm for membrane-organizing and -shaping scaffolds. FEBS Lett. 2006;580(23):5559-5564.

13. Shibata Y, et al. The reticulon and DP1/Yop1p proteins form immobile oligomers in the tubular endoplasmic reticulum. J Biol Chem. 2008; 283(27):18892-18904.

14. $\mathrm{Hu} \mathrm{J}$, et al. Membrane proteins of the endoplasmic reticulum induce high-curvature tubules. Science. 2008;319(5867):1247-1250.

15. Shibata Y, Shemesh T, Prinz WA, Palazzo AF, Kozlov MM, Rapoport TA. Mechanisms determining the morphology of the peripheral ER. Cell. 2010;143(5):774-788.

16. Voeltz GK, Prinz WA, Shibata Y, Rist JM, Rapoport TA. A class of membrane proteins shaping the tubular endoplasmic reticulum. Cell. 2006; 124(3):573-586.

17. Zurek N, Sparks L, Voeltz G. Reticulon short hairpin transmembrane domains are used to shape ER tubules. Traffic. 2011;12(1):28-41.

18. Dawson TR, Lazarus MD, Hetzer MW, Wente SR.
ER membrane-bending proteins are necessary for de novo nuclear pore formation. J Cell Biol. 2009; 184(5):659-675.

19. Ferreirinha F, et al. Axonal degeneration in paraplegin-deficient mice is associated with abnormal mitochondria and impairment of axonal transport. J Clin Invest. 2004;113(2):231-242.

20. Pirozzi M, et al. Intramuscular viral delivery of paraplegin rescues peripheral axonopathy in a model of hereditary spastic paraplegia. J Clin Invest. 2006;116(1):202-208.

21. Beetz C, et al. Exome sequencing identifies a REEP1 mutation involved in distal hereditary motor neuropathy type V. Am J Hum Genet. 2012;91(1):139-145.

22. Azzouz M, Leclerc N, Gurney M, Warter JM, Poindron P, Borg J. Progressive motor neuron impairment in an animal model of familial amyotrophic lateral sclerosis. Muscle Nerve. 1997;20(1):45-51.

23. Saito H, Kubota M, Roberts RW, Chi Q, Matsunami H. RTP family members induce functional expression of mammalian odorant receptors. Cell. 2004;119(5):679-691.

24. Wong DH, Brodsky FM. 100-kD proteins of Golgiand trans-Golgi network-associated coated vesicles have related but distinct membrane binding properties. J Cell Biol. 1992;117(6):1171-1179.

25. Hazan J, et al. Spastin, a new AAA protein, is altered 
in the most frequent form of autosomal dominant spastic paraplegia. Nat Genet. 1999;23(3):296-303.

26. Fink JK. Hereditary spastic paraplegia. Curr Neurol Neurosci Rep. 2006;6(1):65-76.

27. Shibata Y, Hu J, Kozlov MM, Rapoport TA. Mechanisms shaping the membranes of cellular organelles. Annu Rev Cell Dev Biol. 2009;25:329-354.

28. Friedman JR, Voeltz GK. The ER in 3D: a multifunctional dynamic membrane network. Trends Cell Biol. 2011;21(12):709-717.

29. O'Sullivan NC, Jahn TR, Reid E, O'Kane CJ. Reticulon-like-1, the Drosophila orthologue of the hereditary spastic paraplegia gene reticulon 2 , is required for organization of endoplasmic reticulum and of distal motor axons. Hum Mol Genet. 2012; 21(15):3356-3365.

30. Hu J, et al. A class of dynamin-like GTPases involved in the generation of the tubular ER network. Cell. 2009;138(3):549-561.

31. Orso G, et al. Homotypic fusion of ER membranes requires the dynamin-like GTPase atlastin. Nature. 2009;460(7258):978-983.

32. Montenegro G, et al. Mutations in the ER-shaping protein reticulon 2 cause the axon-degenerative disorder hereditary spastic paraplegia type 12. J Clin Invest. 2012;122(2):538-544.

33. Alvarez V, et al. Mutational spectrum of the SPG4 (SPAST) and SPG3A (ATL1) genes in Spanish patients with hereditary spastic paraplegia. BMCNewrol. 2010;10:89.

34. Hentschke $\mathrm{M}$, et al. Mice with a targeted disruption of the $\mathrm{Cl}^{-} / \mathrm{HCO}_{3}{ }^{-}$exchanger AE3 display a reduced seizure threshold. Mol Cell Biol. 2006;26(1):182-191.

35. Schwintzer L, Koch N, Ahuja R, Grimm J, Kessels MM, Qualmann B. The functions of the actin nucleator Cobl in cellular morphogenesis critically depend on syndapin I. EMBOJ. 2011;30(15):3147-3159.

36. Irintchev A, Simova O, Eberhardt KA, Morellini F, Schachner M. Impacts of lesion severity and tyrosine kinase receptor B deficiency on functional outcome of femoral nerve injury assessed by a novel single-frame motion analysis in mice. EurJ Neurosci. 2005;22(4):802-808.

37. Apostolova I, Irintchev A, Schachner M. Tenascin-R restricts posttraumatic remodeling of motoneuron innervation and functional recovery after spinal cord injury in adult mice. J Neurosci. 2006; 26(30):7849-7859.

38. Poet $\mathrm{M}$, et al. Lysosomal storage disease upon disruption of the neuronal chloride transport protein ClC-6. Proc Natl Acad Sci U S A. 2006;103(37):13854-13859.

39. Hubner CA, Lorke DE, Hermans-Borgmeyer I. Expression of the Na-K-2Cl-cotransporter NKCC1 during mouse development. Mech Dev. 2001; 102(1-2):267-269.

40. Karle KN, Mockel D, Reid E, Schols L. Axonal transport deficit in a KIF5 $\mathrm{A}^{-/-}$mouse model. Nenrogenetics. 2012;13(2):169-179.

41. Sinning A, Liebmann L, Kougioumtzes A, Westermann M, Bruehl C, Hubner CA. Synaptic glutamate release is modulated by the $\mathrm{Na}^{+}$-driven $\mathrm{Cl}^{-} / \mathrm{HCO}_{3}{ }^{-}$ exchanger Slc4a8. J Neurosci. 2011;31(20):7300-7311.

42. Koch N, Dharmalingam E, Westermann M, Qualmann B, Thomas U, Kessels MM. Abp1 utilizes the Arp2/3 complex activator Scar/WAVE in bristle development. J Cell Sci. 2012;125(15):3578-3589.

43. Reeves JP, Dowben RM. Formation and properties of thin-walled phospholipid vesicles. J Cell Physiol. 1969;73(1):49-60 